\title{
SUNLIGHT DRIVEN PHOTOCATALYTIC DEGRADATION OF 2-CHLOROPHENOL BY POLYPYRROLE/ GRAPHENE OXIDE COMPOSITES
}

\author{
Siti Nor Atika Baharin ${ }^{* 1 a}$, Kavirajaa Pandian Sambasevam ${ }^{2 a}$, Nur Farahin Suhaimi ${ }^{3 a}$, \\ A. Arifutzzaman ${ }^{4 b}$, Saidur Rahman ${ }^{5 b}$ and Shafira Nadira Musiran ${ }^{6 a}$
}

\begin{abstract}
${ }^{a}$ School of Chemistry and Environment, Faculty of Applied Sciences, Universiti Teknologi MARA, Cawangan Negeri Sembilan, Kampus Kuala Pilah, 72000, Negeri Sembilan, MALAYSIA. Email: atikabaharin@uitm.edu.my; kavirajaa@uitm.edu.my ${ }^{2}$; farahin2703@gmail.com ${ }^{3}$; shafiranadira96@gmail.com ${ }^{6}$

${ }^{b}$ Research Centre for Nano-Materials and Energy Technology (RCNMET), School of Engineering and Technology, Sunway University, Bandar Sunway, 47500 Selangor, MALAYSIA. Email: rahat.meengg@gmail.com ${ }^{4}$; saidur@sunway.edu.my ${ }^{5}$

Corresponding author: atikabaharin@uitm.edu.my
\end{abstract}

Received: $19^{\text {th }}$ Jun $2020 \quad$ Accepted: $15^{\text {th }}$ Oct 2020

Published: $28^{\text {th }}$ February 2021

DOI: https://doi.org/10.22452/mjs.vol40no1.6

\begin{abstract}
Polypyrrole/graphene oxide (PPy/GO) was synthesised and evaluated as an effective photocatalyst to degrade 2-chlorophenol from aqueous solution under solar light irradiation. The PPy/GO composite was synthesised by the incorporation of PPy with GO using Hummer's method(s). Three different compositions of PPy/GO composites were prepared with a varying ratio of PPy and GO (1:1, 1:4, and 9:1). The physicochemical properties of PPy, GO, and the synthesised PPy/GO composites were characterised using FTIR, UV-Vis, XRD, and SEM with EDX. The results revealed that PPy was successfully integrated with GO forming PPy/GO composites. The photodegradation of $10 \mathrm{ppm}$ of 2-chlorophenol has been observed under sunlight for 3 hours with the exposure contact time of 180 minutes before being tested with UV-Vis in order to obtain the percentage of degradation in the sunlight. The degradation study demonstrates that PPy/GO composite with the ratio of 1:1 exhibited the highest percentage of degradation with $\sim 71.08 \%$ at 180 minutes and $68.96 \%$ at 3 hours.
\end{abstract}

Keywords: Polypyrrole (PPy), Graphene Oxide (GO), Conducting Polymers (CPs), Metal Oxides, Photocatalytic Degradation.

\section{INTRODUCTION}

Photocatalysis is an advanced oxidation process that is also known as 'artificial photosynthesis' to break down the organic and inorganic pollutants for environmental recovery. The utilisation of radiation source and a catalyst is crucial to degrade the pollutants efficiently with the use of green technology. This is a type of technology that converts photonic energy from UV light irradiation to chemical energy using semiconductors as the photocatalysts (BustosRamirez et al., 2015). In other words, semiconductor material will be activated by photons from irradiation with sufficient energy that matches or higher than the bandgap energy between the conduction band and valence band of the material (Igbinosa et al., 2013). 
The organic pollutants, specifically phenolic compounds such as 2-chlorophenol (2-CP) are considered as the most important pollutants that are present in the environment (Rashid et al., 2018). This toxic pollutant has adverse effects on living organisms whereby it has a long-life span in the environment at high toxicity levels. Exposure to chlorophenols has been associated with industrial productions of insecticides, pesticides, pharmaceuticals, and refineries. Occupational exposures through inhalation or penetration through the skin often resulted in asthma, lung cancer, nonHodgkin's lymphoma, and sarcoma. Therefore, in-depth analysis and material development becoming important for effective removal of phenolic compounds from the water body (Sani et al., 2018). Over a decade, several available techniques have been developed such as oxidation via electrochemical, reduction-oxidation approach, membrane filtration, and photocatalytic degradation as the most efficient method to degrade the phenolic compounds for water.

Polypyrrole (PPy) has been widely employed in water remediation due to the properties such as high stability, high charge carriers, good redox activity, strong binding sites, non-toxicity, and most importantly facile synthesis route (Ferenets \& Harlin, 2007). Furthermore, the presence of a positive charge on the PPy backbone makes it a potential adsorbent in photocatalysis reaction (Majumdar et al., 2018). Table 1 shows the hybrid PPy/inorganic composites that were effectively utilised as photocatalyst in wastewater remediation such as $\mathrm{PPy} / \mathrm{TiO}_{2}, \mathrm{PPy} / \mathrm{CdS}$, $\mathrm{PPy} / \mathrm{AgCl}$, and $\mathrm{PPy} / \mathrm{Bi}_{2} \mathrm{O}_{2} \mathrm{CO}_{3}$ (Yuan et al., 2019). However, PPy has few drawbacks such as low in mechanical properties, cumbersome processability, possesses low visible-light absorption range, and inadequate electron transferability for photocatalytic activity (Mitra et al., 2019). Therefore, researchers started to venture into PPy composites with carbon nanomaterial to overcome these drawbacks that will lead to efficient photocatalytic degradation. This is achieved by designing integrated material with a synergetic effect containing PPy and GO resulting in high degradation efficiency, reusability, and steady (Zhao et al., 2019).

Table 1. Photocatalyst PPy-based

\begin{tabular}{ccc}
\hline Photocatalyst & Pollutant & Source of Light \\
\hline $\mathrm{PPy} / \mathrm{TiO}_{2}$ & Methyl Orange & Sunlight \\
\hline $\mathrm{PPy} / \mathrm{CdS}$ & Methylene blue/Rhodamine & Ultra-violet \\
\hline $\mathrm{PPy} / \mathrm{AgCl}$ & Methyl Orange & Sunlight \\
\hline $\mathrm{PPy} / \mathrm{Bi}_{2} \mathrm{O}_{2} \mathrm{CO}_{3}$ & Rhodamine B & Ultra-violet \\
\hline
\end{tabular}

Graphene oxide (GO) has gained worldwide attention due to its mechanical strength, excellent electrical properties such as high electron mobility, chemically stable, good optical properties, and high surface area (Bustos-Ramirez et al., 2015). Besides that, the existence of hydroxyl groups in the GO shows photocatalytic properties as shown by water splitting and hydrogen production (Munawaroh et al., 2018). The presence of hydroxyl, epoxy, carbonyl, and carboxyl groups on GO's surface with high negative charge makes it extremely active (Yu et al., 2019). This shows that the surface of GO can 
be fabricated with a specific functional group such as inorganic particles, polymers, or biomolecules to improve its performance as photocatalyst. GO is a promising agent to improve the physicochemical properties of polymers by forming interfacial bonding with a polymer matrix (Lü et al., 2012). Furthermore, GO is the substance of choice for visible light photocatalysis flourished with $\mathrm{sp}^{2}$ hybridised carbon honeycomb structure with delocalised $\pi$-electron clouds (Chougule et al., 2011). This study aims to benefit from the combination of GO and PPy in terms of the structural, optical, and photocatalytic properties for the remediation of wastewater. The photodegradation techniques conducted with sunlight irradiation were used since it is pollution-free, economical, and good performance in degrading the 2-chlorophenol.

This study aims to integrate PPy with GO via in-situ polymerisation method. The resulted composites were optimised and effectively utilised as a photocatalyst to degrade 2-chlorophenol using direct sunlight radiation.

\section{MATERIALS AND METHODS}

\subsection{Materials}

2-chlorophenol was purchased from Merck Sdn. Bhd., pyrrole (99\%) was obtained from Sigma Aldrich, ammonium persulfate (APS), hydrogen peroxide solution $35 \%$ $\left(\mathrm{H}_{2} \mathrm{O}_{2}\right)$, concentrated sulfuric acid $98 \%$ $\left(\mathrm{H}_{2} \mathrm{SO}_{4}\right)$, hydrochloric acid $(\mathrm{HCl})$, and ethanol were purchased from R\&M Chemicals. Graphite powder was obtained from $\mathrm{HmbG}$ Chemical (Germany), sodium nitrate $\left(\mathrm{NaNO}_{3}\right)$, and potassium permanganate $\left(\mathrm{KMNO}_{4}\right)$ were supplied by Bendosen Chemical (Malaysia). Finally, deionised water was supplied from Purite machine model Fusion system (United
Kingdom) that has ultrapure water $18.2 \mathrm{M} \Omega \mathrm{cm}$ at a flow rate of $48 \mathrm{~L} / \mathrm{hr}$.

\subsection{Preparation of GO}

GO was produced via modified Hummer's method. Typically, graphite flakes $(1 \mathrm{~g})$ and $\mathrm{NaNO}_{3}(0.5 \mathrm{~g})$ were added into a concentrated $\mathrm{H}_{2} \mathrm{SO}_{4}$ in $250 \mathrm{ml}$ conical flask. The solution was then stirred at $600 \mathrm{rpm}$ for 4 hours at $\sim{ }^{\circ} \mathrm{C}$ in an ice bath and $3 \mathrm{~g}$ of $\mathrm{KMnO}_{4}$ was added slowly to the solution. The mixture was stirred at $600 \mathrm{rpm}$ for an hour at $35{ }^{\circ} \mathrm{C}$ and cooled at an ambient temperature. Next, $45 \mathrm{ml}$ of deionised water was added to the mixture in a dropwise technique and stirred for an hour at $600 \mathrm{rpm}$. The solution was heated at $90{ }^{\circ} \mathrm{C}$ for 2 hours and subsequently washed via filtration technique with $100 \mathrm{ml}$ of $5 \% \mathrm{HCl}$ to remove the residues from the oxidants and metal impurities. About $10 \mathrm{ml}$ of $\mathrm{H}_{2} \mathrm{O}_{2}(30 \%)$ was poured to terminate the reaction and stirred at $600 \mathrm{rpm}$ ) for 30 minutes. The mixture was washed three times until the $\mathrm{pH}$ becomes neutral. Then, the solution was centrifuged for 10 minutes at $5000 \mathrm{rpm}$ for purification purposes in which the supernatant was decanted from GO. The product was dried at $60{ }^{\circ} \mathrm{C}$ for 3 hours. Lastly, the product was ground until it became a powder form using a mortar. The powder was washed with deionized water to remove impurities and dried at $60{ }^{\circ} \mathrm{C}$ for 2 hours.

\subsection{Preparation of PPy/GO}

The synthesised GO was added into $50 \mathrm{ml}$ of $1 \mathrm{M} \mathrm{HCl}$ under ultrasonication (Elmasonic, $\mathrm{S} 15 \mathrm{H}$ ) for $30 \mathrm{~min}$ to obtain $\mathrm{GO}$ in a nanometer-scale. Py (monomer) was added in a ratio of GO/Py $(9: 1,1: 1$, and $1: 4)$ into the solution at $0-5{ }^{\circ} \mathrm{C}$ using an ice bath. The solution was stirred for $10 \mathrm{~min}$ at $600 \mathrm{rpm}$ to obtain a well-dispersed suspension. Then, APS was added gradually in the $\mathrm{HCl}$ solution. The 
polymerisation was continued by stirring the solution for 24 hours at $0-5{ }^{\circ} \mathrm{C}$ with a constant speed. The $\mathrm{PPy} / \mathrm{GO}$ precipitates were centrifuged at $5000 \mathrm{rpm}$ and rinsed with acetone to remove the unreacted monomers and oligomers several times until the filtrate became colourless. The precipitate was then extracted using vacuum suction and dried at $70{ }^{\circ} \mathrm{C}$ for 12 hours.

\subsection{Characterisations}

Fourier transform infrared (FT-IR) spectroscopy was recorded using a PerkinElmer FT-IR between $4000-400 \mathrm{~cm}^{-1}$ (Perkin Elmer, Malaysia). The samples were pressed by ATR crystal and the spectra of the samples were recorded. UV-Vis solid (Perkin Elmer Lambda 750) was used to record the powder sample in the range of 200-800 $\mathrm{nm}$. PPy powder was placed at the quartz spheres in front of the incident light window and subjected to light reflection from barium sulfate-coated detector. The procedure was repeated for GO and PPy/GO (1:1, 9:1, and 1:4) composites samples. Analysis of x-ray diffractometry (XRD) was performed using Panalytical Model Empyrean at $40 \mathrm{kV}$ and $35 \mathrm{~mA}$ using $\mathrm{Cu}, \mathrm{K} \alpha$ radiation $(\ddot{\mathrm{y}}=1.54059 \AA)$ (Panalytical, Malaysia) to determine the crystallographic phase of PPy/GO composites. PPy powder was placed on the sample holder at the sample surface. The sample was smeared uniformly on the glass surface. The procedure was repeated for GO and PPy/GO (1:1, 9:1, and 1:4) composites samples.

\subsection{Degradation Studies - Screening Process}

10 ppm 2-chlorophenol (20 ml) was prepared and placed in 6 units the beaker. The first beaker contains $10 \mathrm{ppm}$ of 2-chlorophenol as a blank while 5 other beakers with 10 ppm of 2-chlorophenol were added with $10 \mathrm{mg}$ of PPy, GO and the three different ratio of PPy/GO (1:1, 9:1, and 1:4) composites. Lastly, the mixture was exposed under the sunlight for 3 hours. The absorbance value form UV-Vis $(\lambda=295 \mathrm{~nm})$ was recorded before and after 3 hours of sunlight exposure.

$$
\text { Degradation of } 2-C P(\%)=\frac{A^{\circ}-A t}{A^{\circ}} \times 100 \%
$$

Where $\mathrm{A}^{\circ}$ is the initial absorption of 2-chlorophenol and $A_{t}$ is the absorption of 2-chlorophenol at sampling time during the screening.

\section{RESULTS AND DISCUSSION}

FTIR spectra of PPy, GO, and PPy/GO (1:1) composite is shown in Figure 1. In Figure 1 (a), the N-H stretching of pyrrole ring has been depicted at $3103.17 \mathrm{~cm}^{-1}$. Meanwhile, the other characteristic peaks of pyrrole such as $\mathrm{C}$ $\mathrm{N}, \mathrm{C}=\mathrm{C}$ and $\mathrm{C}-\mathrm{H}$ stretching were ascribed to 1176.18, 1550.40, and $1298 \mathrm{~cm}^{-1}$, respectively (He et al., 2015). Figure 1(b) shows the GO spectrum with a wide peak at $3393.58 \mathrm{~cm}^{-1}$ corresponding to the $\mathrm{O}-\mathrm{H}$ bending at its surface and another peak at $1728.54 \mathrm{~cm}^{-1}$ corresponds to the carbonyl $(\mathrm{C}=\mathrm{O})$ stretching band. Besides that, the carbonyl (C-O) peak also was detected at $1050.17 \mathrm{~cm}^{-1}$ (Cao et al., 2015). Figure 1 (c) spectrum gives an in-depth understanding of the FTIR appearance for PPy/GO (1:1) composite. Spectra for PPy/GO 1:1 appeared at $3366.89 \mathrm{~cm}^{-1}$ attributed to the broad peak of $\mathrm{O}-\mathrm{H}$ on the surface of GO. The increased wave number belongs to the $\mathrm{C}=\mathrm{C}$ stretching vibrations from 1550.40 to $1557.93 \mathrm{~cm}^{-1}$ is due to the interaction of $\pi-\pi$ hydrogen bonds between the GO layer with the aromatic PPy ring. 
In addition, the peak attributed to the $\mathrm{C}=\mathrm{O}$ stretching vibration of the GO/PPy composite downshifts to $1627.31 \mathrm{~cm}^{-1}$ from $1728.54 \mathrm{~cm}^{-1}$ compared to the GO spectra could be due to the interaction of the GO layers and the hydrogen bonding with the aromatic
PPy rings. This indicates that the chemical bonding between the GO layer and the PPy layer, as the $\pi-\pi$ interaction has formed in the composite, has permitted the well-dispersed deposition of the polymer on the GO active surface (Yang et al., 2011).

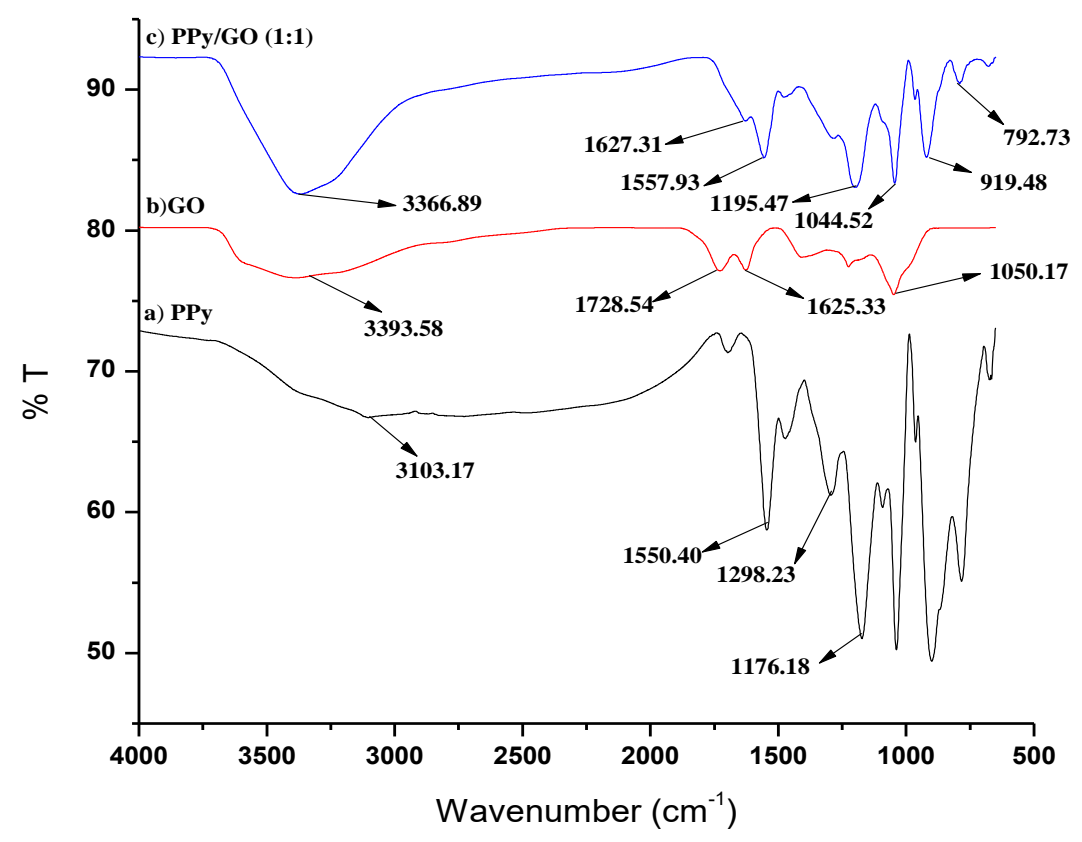

Figure 1. FTIR absorption spectra of a) PPy, b) GO, c) PPy/GO (1:1) composites

Figure 2 shows the FTIR characteristic peaks of PPy/GO composites. PPy/GO 1:1 and 1:4 exhibited a broad peak in the range of 3600 $-2900 \mathrm{~cm}^{-1}$ that includes $\mathrm{N}-\mathrm{H}, \mathrm{OH}, \mathrm{CH}_{3}$, and $\mathrm{C}-\mathrm{H}$ stretching peaks. However, this broad peak was greatly suppressed in the PPy/GO (9:1) probably due to the predominating content of PPy (Su et al., 2013). The $\mathrm{C}=\mathrm{O}$ peak that arose from GO was found in PPy/GO (1:4) at $1718 \mathrm{~cm}^{-1}$. The same peak was not observed in the PPy/GO (1:1) suggesting that GO was effectively reduced by the incorporation of $\mathrm{PPy}$. A similar $\mathrm{C}=\mathrm{O}$ was not seen in $\mathrm{PPy} / \mathrm{GO}$
(9:1) probably due to the predominating factor by pristine PPy. A new peak was observed at $1195.47 \mathrm{~cm}^{-1}$ for PPy/GO (1:1) indicating the effective doping of PPy with GO. Some shifts were observed for PPy/GO (9:1) at 1168.30 and $890.57 \mathrm{~cm}^{-1}$ revealing the $\pi-\pi$ synergy between unoxidised GO domain and pyrrole rings of PPy (Yang et al., 2011). Overall, all of the characteristic peaks of PPy and GO were present in the $\mathrm{PPy} / \mathrm{GO}$ composites hence proving the integration of GO in the PPy matrix. 


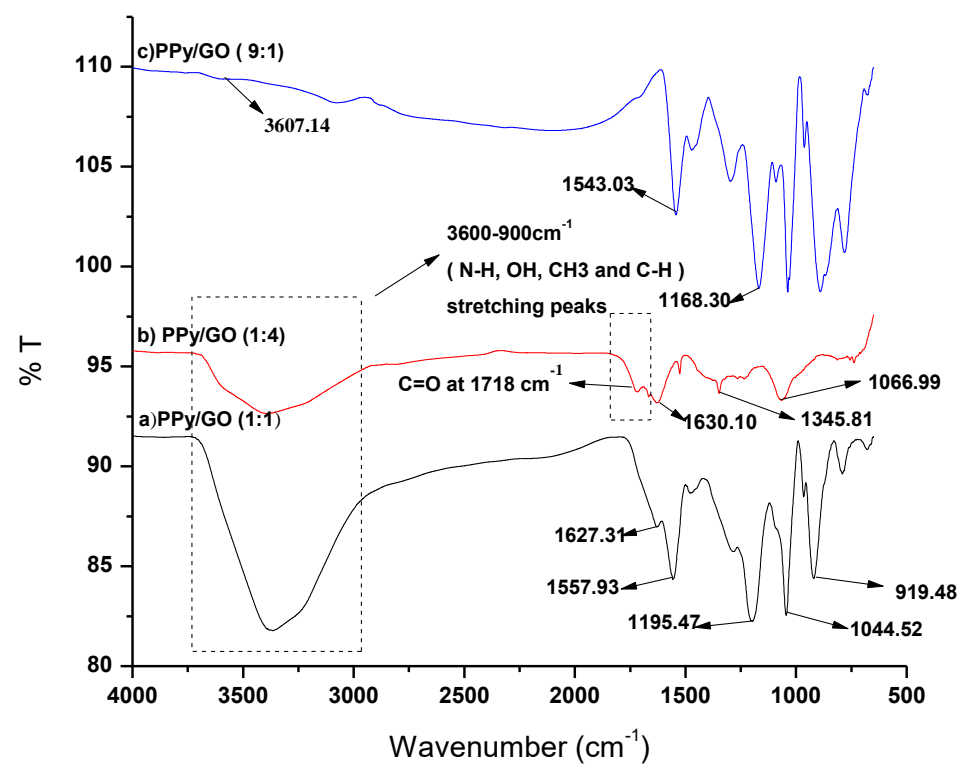

Figure 2. FTIR absorption spectra of a) PPy/GO (1:1) composite, b) PPy/ GO (1:4) composite, and c) $\mathrm{PPy} / \mathrm{GO}(9: 1)$ composite

Figure 3 displays the UV-Vis spectra of PPy, GO, and PPy/GO composites with a wavelength range of 200-800 $\mathrm{nm}$. Three absorption peaks were observed for pristine PPy at $259.85,379$, and $533.98 \mathrm{~nm}$ attributing to the $\pi-\pi^{*}$ and $\pi^{*}$ - polaron and $\pi$ - polaron peaks transitions of PPy, respectively (Emiru $\&$ Ayele, 2017). GO has wide peaks at 273 and $335 \mathrm{~nm}$, corresponding to the $\mathrm{C}=\mathrm{C}$ bonding transition and the $\mathrm{C}=\mathrm{O}$ transition, respectively (Shrikrushna et al., 2015).

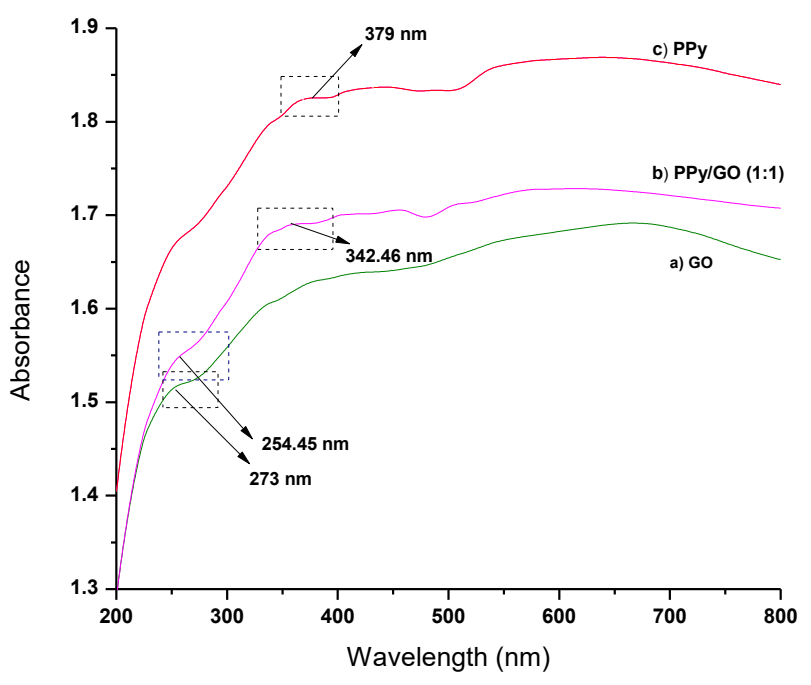

Figure 3. UV-Vis absorption a) GO, b) PPy/GO (1:1) composite, and c) PPy

Figure 4 exhibits the UV-Vis spectra of PPy/GO composites. All PPy and GO peaks features were found in PPy/GO composites. The strong absorption peak of PPy/GO (1:1) and PPy/GO (1:4) is similar to PPy confirming the composite's $\pi-\pi$ interaction between PPy and GO. 


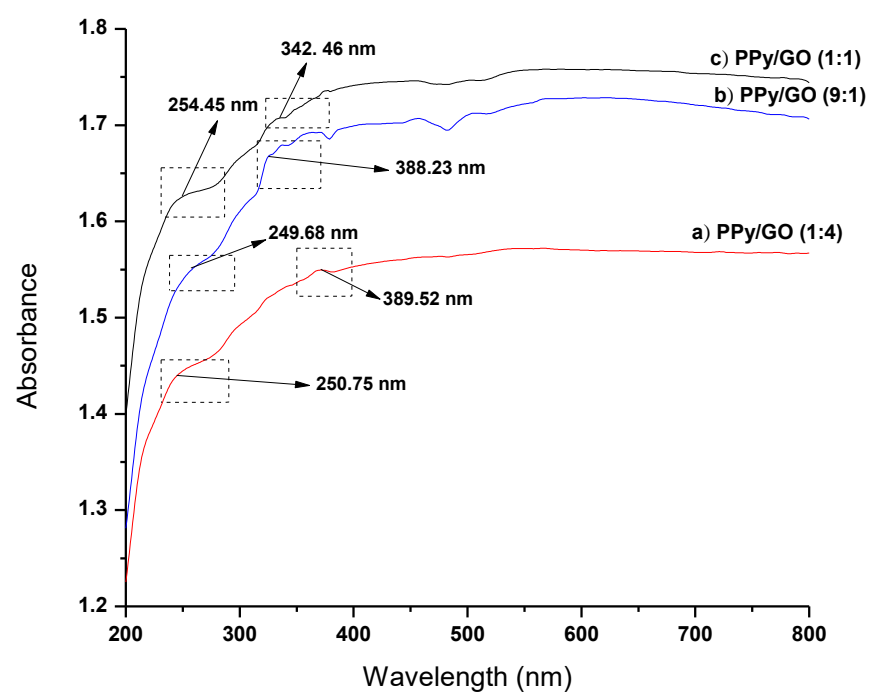

Figure 4. UV-Vis spectra of a) PPy/GO (1:4) composite, b) PPy/GO (9:1) composite, and c) PPy/GO (1:1) composite

Figure 5 exhibits the XRD diffractograms of $\mathrm{PPy}, \mathrm{GO}$, and $\mathrm{PPy} / \mathrm{GO}$ composites. PPy in Figure 5a indicates an amorphous nature with the presence of a broad peak at $2 \Theta=25.89^{\circ}$ (Gascho et al., 2019) in the diffractogram. Meanwhile, GO showed a significant peak at $2 \Theta=9.82^{\circ}$ showing that the use of Hummer's method has successfully synthesised GO with the help of $\mathrm{Mn}_{2} \mathrm{O}_{7}$ as the oxidising agent. This indicates the competence of the oxidation reaction by increasing the interplanar distance between the graphene layers due to the presence of oxygen groups (Wu et al., 2017). The sharp diffraction peak at $2 \theta=9.28^{\circ}$ disappeared for the PPy/GO (1:1) composites. This indicates that GO was successfully reduced by the hydrogen ion from water molecules under the influence of high temperature and high pressure during the hydrothermal process. The integration of GO and PPy were also effectively held by the $\pi-\pi$ and electrostatic attractions (Konwer et al., 2011).

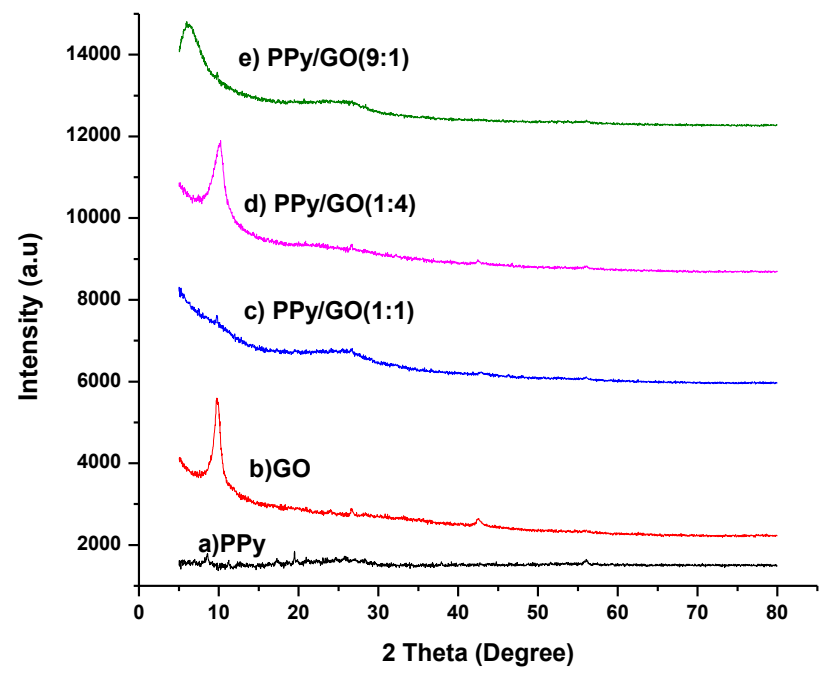

Figure 5. XRD patterns of a) PPy, b) GO, c) PPy/GO (1:1) composite, d) PPy/GO(1:4)composite, and e) PPy/GO (9:1) 
Figure 6 displays the SEM images of PPy, GO, and PPy/GO composite (1:1). The images of PPy (Figure 6a) exhibits a hemispherical and cauliflower-like structure (Yu et al., 2019). Meanwhile, the image of GO in Figure 6b represents layers of flaky shapes (Deng et al., 2011). This is a similar observation reported by $\mathrm{Yu}$ et al., (2019) as seen in Figure 6c revealing PPy/GO films with a non-uniform surface with flaky lumps morphology and half embedded onto the polymer matrix. This indicates the incorporation of GO sheets is formed due to the unevenness of the PPy films (Molina et al., 2015). This also proves the existence of both $\mathrm{GO}$ and PPy structure in the PPy/GO composite. In addition, the flaky structure of GO predominates the PPy structure, which also resulted in strong peaks that are seen from the diffractograms in Figure 5 (c-e).
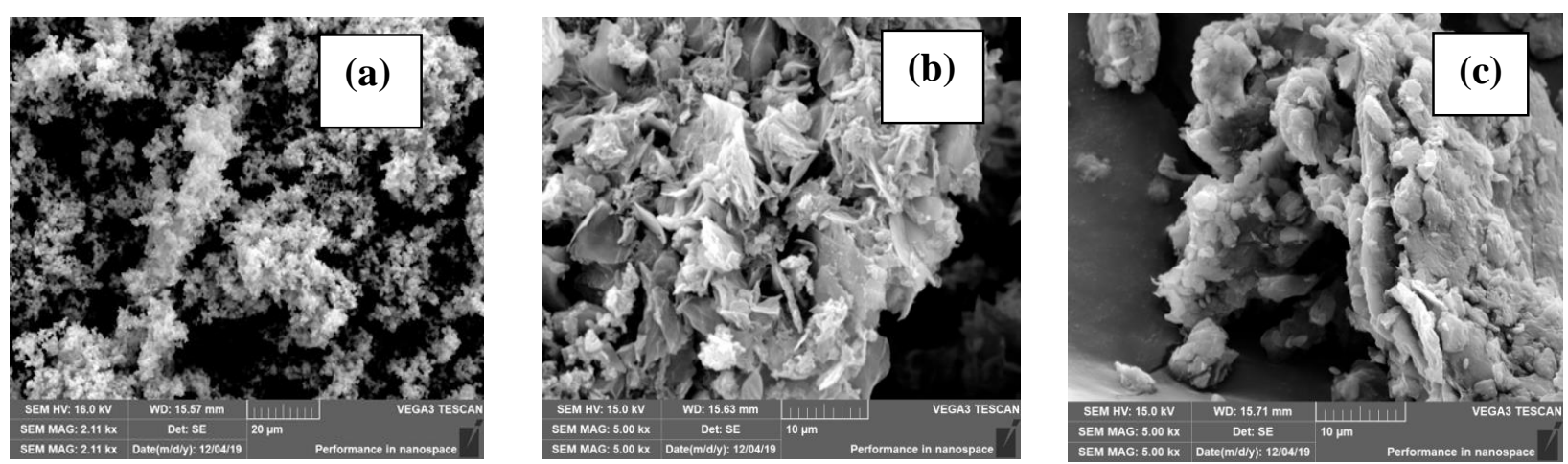

Figure 6. SEM images of a) PPy, b) GO, and c) PPy/GO

The EDX spectral analysis of PPy, GO, and PPy/GO composites are shown and represented in Figure 7 and Table 1. The analysis confirms the existence of the most remarkable elements of $\mathrm{C}, \mathrm{N}$, and $\mathrm{O}$ and the quantitative elemental composition. EDX analysis also confirmed that $\mathrm{PPy} / \mathrm{GO}$ composite contain higher wt $\%$ of carbon 53.64 since it is present in both PPy and GO. The carbon is the results from pyrrole $\left(\mathrm{C}_{4} \mathrm{H}_{5} \mathrm{~N}\right)$ and $\mathrm{GO}$ while nitrogen belongs to the pyrrole units and oxygen are predominantly from GO (Tseng et al., 2017).
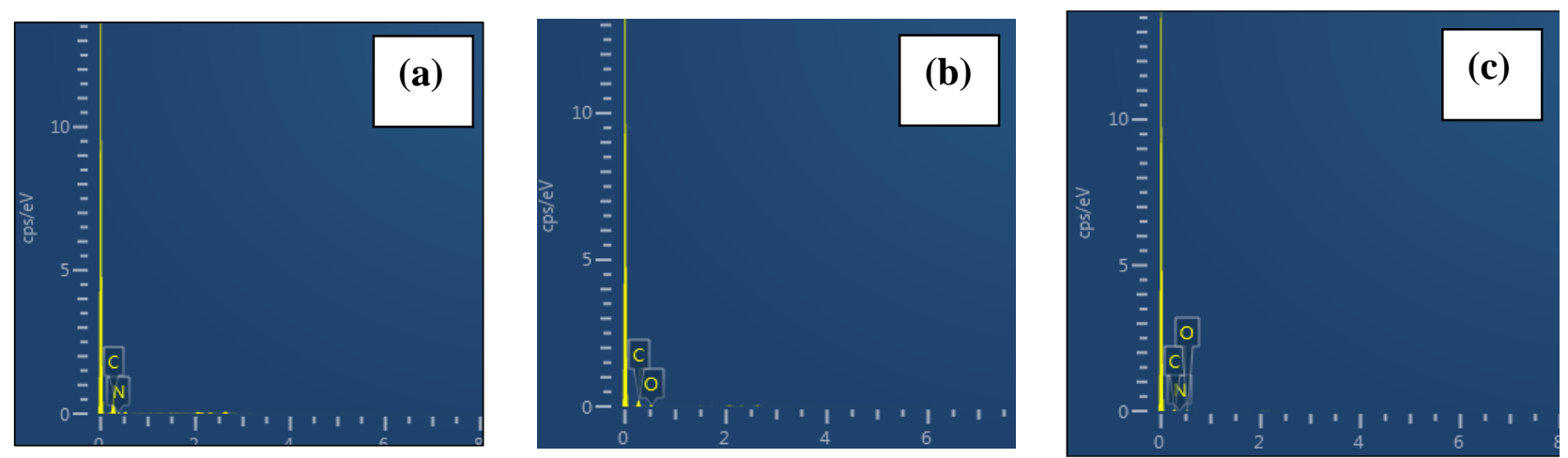

Figure 7. EDX Spectra a) PPy, b) GO, and c) PPy/GO (1:1) composite 
Table 1. EDX analysis of PPy, GO, and PPy/GO

\begin{tabular}{cccc}
\hline \multirow{2}{*}{ Element $(\mathbf{w t} \%)$} & \multicolumn{3}{c}{ Sample } \\
\cline { 2 - 4 } & PPy & GO & PPy/GO \\
\hline $\mathrm{C}$ & 63.16 & 71.80 & 53.64 \\
\hline $\mathrm{N}$ & 36.84 & - & 11.56 \\
\hline $\mathrm{O}$ & - & 28.20 & 34.81 \\
\hline
\end{tabular}

Figure 8 illustrates the photocatalytic activities of PPy, GO, and PPy/GO composites against $10 \mathrm{ppm}$ of 2-chlorophenol in an aqueous solution under sunlight. It can be seen that PPy/GO (1:1) exhibits the highest egradation ( $\mathrm{Li}$ et al., 2012). This correlates with the ability of PPy/GO in inhibiting the hole recombination and increasing the degradation efficiency (Naknikham et al., 2019). In contrast, PPy/GO (1:4) and PPy/GO
(9:1) showed the lowest degradation due to partial shielding of the sunlight radiation thus, limiting the activation of the PPy surface by the light beam. Besides that, no new interface was created with PPy under GO loading however, a flaky structure was formed with PPy that can hinder electron-hole recombination (Alshabanat \& Al-Anazy, 2018).

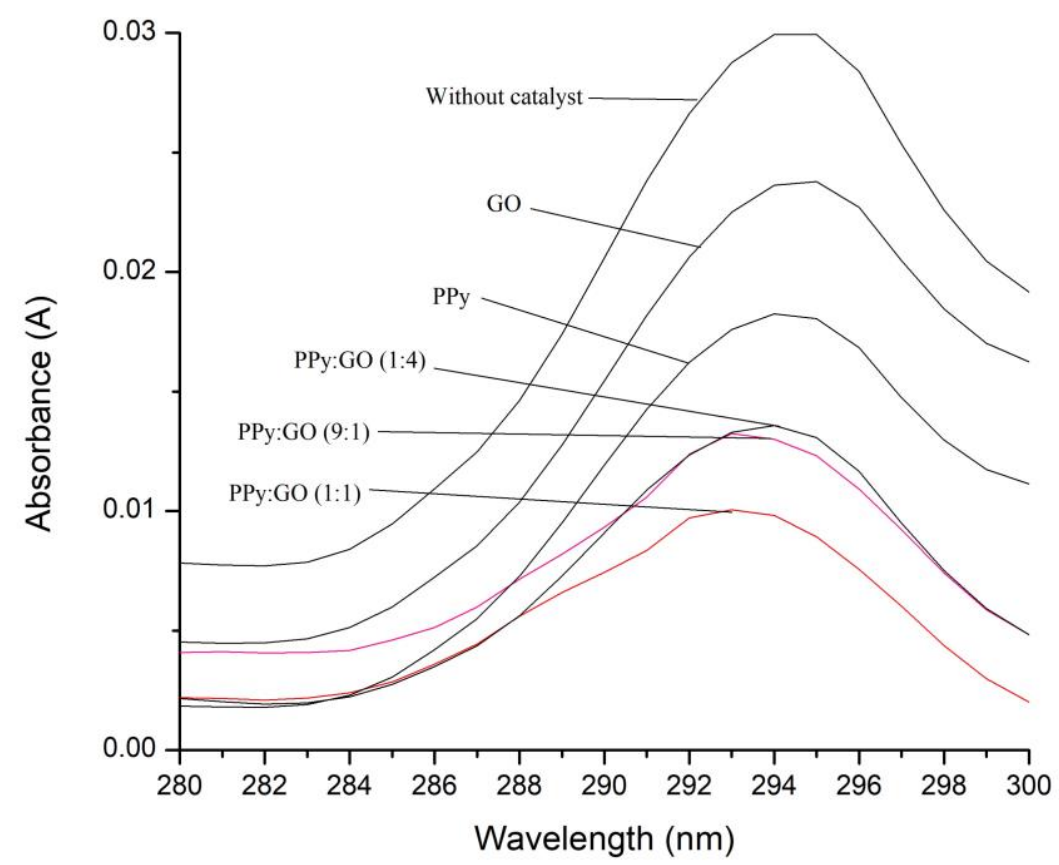

Figure 8. 2-chlorophenol $\%$ of degradation under direct sunlight (condition: $0.01 \mathrm{~g}$ composite, 20 $\mathrm{mL}$ of $10 \mathrm{ppm}$ of 2-chlorophenol were exposed to the sunlight for 180 minutes)

Therefore, PPy/GO (1:1) was selected and subjected to different contact time with direct sunlight in the photodegradation of 2chlorophenol. As seen in Figure 9, the highest percentage of degradation was $71.85 \%$ at 180 minutes. This proves that $180 \mathrm{~min}$ is the effective time for the irradiation exposure to sunlight. This is due to the improvement of the 
excitation process of the electron $\left(\mathrm{e}^{-}\right)$and $\mathrm{H}^{+}$in the photocatalyst that influences the penetration of the free radical against 2chlorophenol (Shahabuddin et al., 2016). Figure 10 illustrates the photogenerated electrons in the conduction band (CB) of PPy would migrate to $\mathrm{GO}$ while the holes in the valence band (VB) of PPy could transfer to GO. As a result, the recombination of the formed electrons and holes are prevented and efficiently degrading 2-chlorophenol (Valdivel et al., 2016).

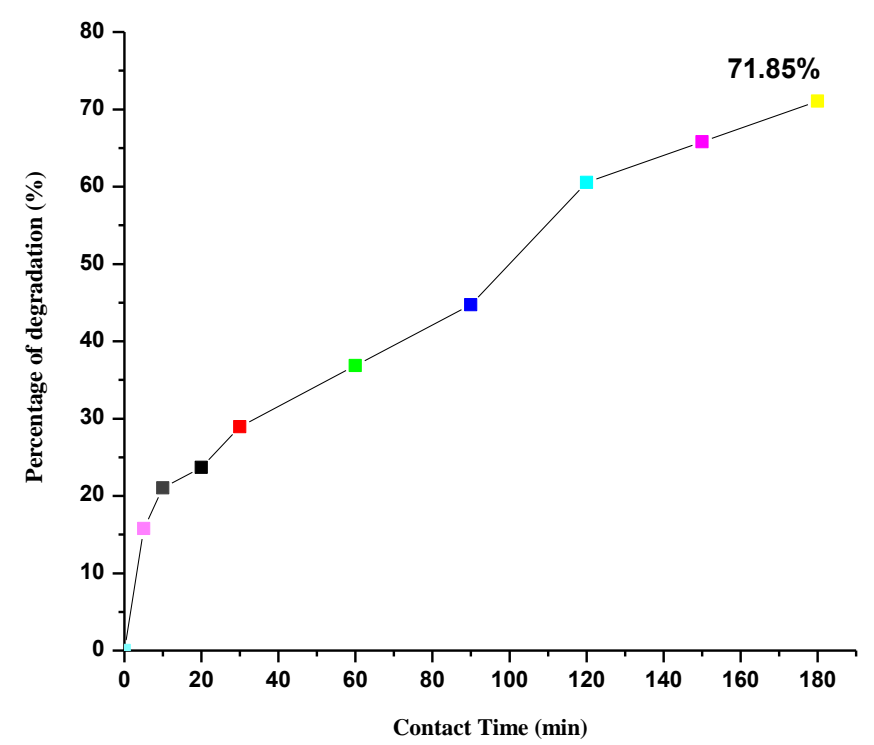

Figure 9. Percentage degradation efficiency on different contact time (condition: $0.01 \mathrm{~g}$ composite, $20 \mathrm{~mL}$ of $10 \mathrm{ppm}$ 2-chlorophenol)

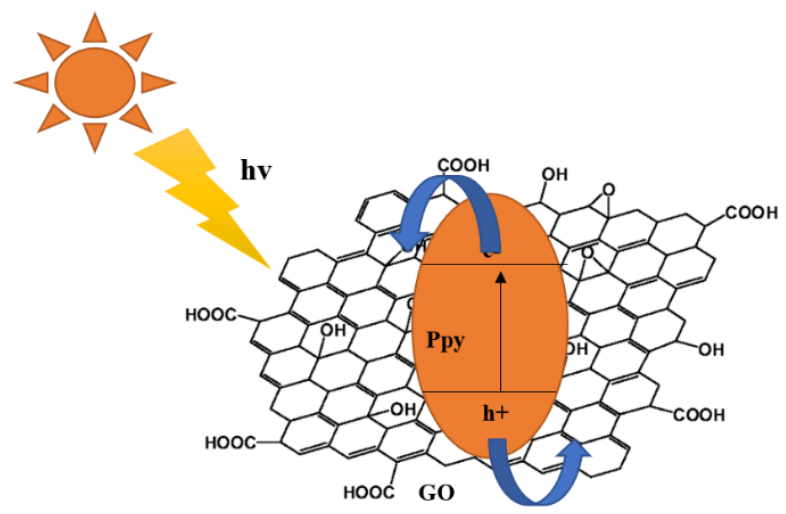

Figure 10. A schematic diagram of photogenerated electrons/hole in the PPy/GO composites

\section{CONCLUSIONS}

In conclusion, PPy was successfully synthesised in the presence of GO under chemical oxidative method. The characterisations revealed that the optimum amount of GO possessed greater influence on
PPy's photocatalytic properties. PPy/GO (1:1) showed the highest photocatalytic activity among the other composites in 2-chlorophenol degradation at $10 \mathrm{ppm}$. Furthermore, exposure to sunlight in different contact time from 5 $180 \mathrm{~min}$ shows that $180 \mathrm{~min}$ is the best degradation $(71.05 \%)$. In general, it has been 
proven that GO can be integrated into PANI matrix to improve the photocatalytic degradation of 2-CP.

\section{ACKNOWLEDGEMENT}

We would like to acknowledge that this project received funding from the Ministry of Higher Education [FRGS/1/2018/STG01/ UITM/02/1] and Geran SDG Lestari [600RMC/LESTARI SDG-T 5/3 (080/2019)] by Universiti Teknologi MARA.

\section{REFERENCES}

Alshabanat, M. N., \& Al-Anazy, M. M. (2018). An Experimental Study of Photocatalytic Degradation of Congo Red Using Polymer Nanocomposite Films. Journal of Chemistry, 2018, 9651850 (18). https://doi.org/10.1155/2018/9651850

Bustos-Ramirez, K., Barrera-Diaz, C. E., De Icaza, M., Martínez-Hernández, A. L., \& Velasco-Santos, C. (2015) Photocatalytic activity in phenol removal of water from graphite and graphene oxides: Effect of degassing and chemical oxidation in the synthesis process. Journal of Chemistry, 2015, 1-10. https://doi.org/10.1155/2015/254631

Cao, J., Wang, Y., Chen, J., Li, X., Walsh, F. C., Ouyang, J. H., ... Zhou, Y. (2015). Three-dimensional graphene oxide/polypyrrole composite electrodes fabricated by one-step electrodeposition for high performance supercapacitors. Journal of Materials Chemistry A, 3(27), 14445-14457. https://doi.org/10.1039/c5ta02920a

Chougule, M. A., Pawar, S. G., Godse, P. R., Mulik, R. N., Sen, S., \& Patil, V. B. (2011). Synthesis and Characterization of
Polypyrrole (PPy) Thin Films. Soft Nanoscience Letters, 01(01), 6-10. https://doi.org/10.4236/snl.2011.11002

Deng, M., Yang, X., Silke, M., Qiu, W., Xu, M., Borghs, G., \& Chen, H. (2011). Electrochemical deposition of polypyrrole/graphene oxide composite on microelectrodes towards tuning the electrochemical properties of neural probes. Sensors and Actuators B: Chemical, 158(1), 176-184. https://doi.org/10.1016/J.SNB.2011.05.0 62

Emiru, T. F., \& Ayele, D. W. (2017). Controlled synthesis, characterization and reduction of graphene oxide: A convenient method for large scale production. Egyptian Journal of Basic and Applied Sciences, 4(1), 74-79. https://doi.org/10.1016/j.ejbas.2016.11.0 02

Ferenets, M., \& Harlin, A. (2007). Chemical in situ polymerization of polypyrrole on poly(methyl metacrylate) substrate. Thin Solid Films, 515(13), 5324-5328. https://doi.org/10.1016/j.tsf.2007.01.008

Gascho, J. L. S., Costa, S. F., Recco, A. A. C., \& Pezzin, S. H. (2019). Graphene Oxide Films Obtained by Vacuum Filtration: XRay Diffraction Evidence of Crystalline Reorganization. Journal of Nanomaterials, 2019, 5963148 (1-12). https://doi.org/10.1155/2019/5963148

He, D., Peng, Z., Gong, W., Luo, Y., Zhao, P., \& Kong, L. (2015). Mechanism of a green graphene oxide reduction with reusable potassium carbonate. RSC Advances, 5(16), 11966-11972. https://doi.org/10.1039/c4ra14511a

Igbinosa, E. O., Odjadjare, E. E., Chigor, V. N., Igbinosa, I. H., Emoghene, A. O., Ekhaise, F. O., ... Montoliu, C. (2013). Toxicological Profile of Chlorophenols 
and Their Derivatives in the Environment: The Public Health Perspective. The Scientific World Journal, 2013, 11. https://doi.org/10.1155/2013/460215

Konwer, S., Boruah, R., \& Dolui, S. K. (2011). Studies on conducting polypyrrole/graphene oxide composites as supercapacitor electrode. Journal of Electronic Materials, 40(11), 2248-2255. https://doi.org/10.1007/s11664-0111749-z

Li, S., Lu, X., Xue, Y., Lei, J., Zheng, T., \& Wang, C. (2012). Fabrication of Polypyrrole/Graphene Oxide Composite Nanosheets and Their Applications for $\mathrm{Cr}(\mathrm{VI})$ Removal in Aqueous Solution. PLoS ONE, 7(8), e43328. https://doi.org/10.1371/journal.pone.004 3328

Lü, K., Zhao, G., \& Wang, X. (2012). A brief review of graphene-based material synthesis and its application in environmental pollution management. Chinese Science Bulletin, 57(11), 12231234. https://doi.org/10.1007/s11434012-4986-5

Majumdar, S., Nath, J., \& Mahanta, D. (2018). Surface modified polypyrrole for the efficient removal of phenolic compounds from aqueous medium. Journal of Environmental Chemical Engineering, 6(2), 2588-2596. https://doi.org/10.1016/j.jece.2018.04.00 2

Mitra, M., Ahamed, S. T., Ghosh, A., Mondal, A., Kargupta, K., Ganguly, S., \& Banerjee, D. (2019). Polyaniline/Reduced Graphene Oxide Composite-Enhanced Visible-LightDriven Photocatalytic Activity for the Degradation of Organic Dyes. ACS Omega, 4(1), 1623-1635. https://doi.org/10.1021/acsomega.8b029 41

Molina, J., Zille, A., Fernández, J., Souto, A. P., Bonastre, J., \& Cases, F. (2015). Conducting fabrics of polyester coated with polypyrrole and doped with graphene oxide. Synthetic Metals, 204, 110-121.

https://doi.org/10.1016/j.synthmet.2015. 03.014

Munawaroh, H., Sari, P. L., Wahyuningsih, S., \& Ramelan, A. H. (2018). The photocatalytic degradation of methylene blue using graphene oxide $(\mathrm{GO}) / \mathrm{ZnO}$ nanodrums. In AIP Conference Proceedings (Vol. 2014, p. 020119). American Institute of Physics Inc. https://doi.org/10.1063/1.5054523

Naknikham, U. ; Magnacca, G. ;, Qiao, A. ;, Kristensen, P. K., Boffa, V. ; \& Yue, Y. (2019). Phenol Abatement by Titanium Dioxide Photocatalysts: Effect of The Graphene Oxide Loading. Nanomaterials, 9(7), 947 (1-11). https://doi.org/10.3390/nano9070947

Rashid, S., Mazlan, N. A., Sapari, J. M., Raoov Ramachandran, M., \& Pandian Sambasevam, K. (2018). Fabrication of magnetic nanoparticles coated with polyaniline for removal of 2, 4dinitrophenol. Journal of Physics: Conference Series, 1123, 012015. https://doi.org/10.1088/17426596/1123/1/012015

Sani, N. A. N. M., Mazlan, N. A., Mohamed, A. H., Sambasevam, K. P., Jantan, K. A., Ramachandran, M. R., \& Sapari, J. M. (2018). Removal of 2,4-dinitrophenol (2,4-DNP) by using magnetic nanoparticles (MNPs) coated with polypyrrole (PPy). IOP Conference Series: Materials Science and Engineering, 458(1), 012007. 
https://doi.org/10.1088/1757-

899X/458/1/012007

Shahabuddin, S., Muhamad Sarih, N., Mohamad, S., \& Joon Ching, J. (2016). SrTiO3 Nanocube-Doped Polyaniline Nanocomposites with Enhanced Photocatalytic Degradation of Methylene Blue under Visible Light. Polymers, 8(2), 27.

https://doi.org/10.3390/polym8020027

Shrikrushna, S., Kher, J. A., \& Kulkarni, M. V. (2015). Influence of Dodecylbenzene Sulfonic Acid Doping on Structural, Morphological, Electrical and Optical Properties on Polypyrrole/3C-SiC Nanocomposites. J Nanomed Nanotechnol, $\quad 6(5), \quad 313$. https://doi.org/10.4172/2157-

7439.1000313

Su, C., Wang, L., Xu, L., \& Zhang, C. (2013). Synthesis of a novel ferrocene-contained polypyrrole derivative and its performance as a cathode material for Liion batteries. Electrochimica Acta, 104, 302-307.

https://doi.org/10.1016/j.electacta.2013.0 4.127

Tseng, K.-H., Chung, M.-Y., Chang, C.-Y., \& Cheng, T.-S. (2017). A Study of Photocatalysis of Methylene Blue of TiO 2 Fabricated by Electric Spark Discharge Method. Journal of Nanomaterials, 2017, 9346201. https://doi.org/10.1155/2017/9346201

Vadivel, S., Theerthagiri, J., Madhavan, J., \& Maruthamani, D. (2016). Synthesis of polyaniline/graphene oxide composite via ultrasonication method for photocatalytic applications. Materials Focus, 5(4), 393-
397.

https://doi.org/10.1166/mat.2016.1367

Wu, B., Zhang, X., Huang, B., Zhao, Y., Cheng, C., \& Chen, H. (2017). HighPerformance wireless ammonia gas sensors based on reduced graphene oxide and Nano-Silver ink hybrid material loaded on a patch antenna. Sensors (Switzerland), $\quad$ 17(9), 2070. https://doi.org/10.3390/s17092070

Yang, S., Shen, C., Liang, Y., Tong, H., He, W., Shi, X., ... Gao, H. J. (2011). Graphene nanosheets-polypyrrole hybrid material as a highly active catalyst support for formic acid electro-oxidation. Nanoscale, $\quad 3(8), \quad 3277-3284$. https://doi.org/10.1039/c1nr10371g

Yu, F., Bai, X., Yang, C., Xu, L., \& Ma, J. (2019). Reduced Graphene Oxide-P25 Nanocomposites as Efficient Photocatalysts for Degradation of Bisphenol A in Water. Catalysts, 9(7), 607.

https://doi.org/10.3390/catal9070607

Yuan, X., Floresyona, D., Aubert, P. H., Bui, T. T., Remita, S., Ghosh, S., ... Remita, H. (2019). Photocatalytic degradation of organic pollutant with polypyrrole nanostructures under $\mathrm{UV}$ and visible light. Applied Catalysis B: Environmental, 242, 284-292. https://doi.org/10.1016/j.apcatb.2018.10. 002

Zhao, Y., Xia, K., Zhang, Z., Zhu, Z., Guo, Y., \& Qu, Z. (2019). Facile synthesis of polypyrrole-functionalized $\mathrm{CoFe} 2 \mathrm{O} 4$ @SiO 2 for removal for $\mathrm{Hg}(\mathrm{II})$. Nanomaterials, 9(3), 455 (1-21). https://doi.org/10.3390/nano9030455 